\title{
SIGNIFICADOS INSTITUCIONALES DE LA DEMOSTRACIÓN. IMPLICACIONES PARA LA EDUCACIÓN MATEMÁTICA*
}

\author{
GODINO, JUAN D. ${ }^{1}$ y RECIO, ÁNGEL M. \\ ${ }^{1}$ Departamento de Didáctica de la Matemática. Facultad de Educación, Campus de Cartuja \\ 18071 Granada \\ E-mail: jgodino@ugr.es \\ ${ }^{2}$ Universidad de Córdoba. Departamento de Matemáticas e Informática. Facultad de Ciencias \\ de la Educación \\ E-mail: ma1marea@uco.es
}

\begin{abstract}
SUMMARY
In this paper the following main features of the meaning of proof in different institutional contexts, logic and foundations of mathematics, professional mathematics, experimental sciences, daily life and teaching of mathematics are analysed.This analysis suggests that the study of the epistemological and didactical problems involved in the teaching of proof in school mathematics should be carried out within the most general framework of human argumentative practices. A possible explanation for some students' cognitive conflict and difficulties with mathematical proof is the fact that at the different teaching levels and various stages of personal mathematical activity, the institutional meanings of proof are intertwined.
\end{abstract}

\section{INTRODUCCIÓN}

Se observa en los últimos años un interés creciente en educación matemática por la problemática de la enseñanza y aprendizaje de la demostración. La mejor referencia que se puede citar para apoyar esta afirmación es la revista electrónica sobre la demostración editada por Nicolas Balacheff (URL, http://www-cabri.imag.fr/ Preuve/). Este interés parece justificado por el papel esencial de las situaciones y procesos de validación en la propia matemática y el bajo nivel que muestran los estudiantes en la comprensión y elaboración de demostraciones (Fischbein, 1982; Senk, 1985; Martin y Harel, 1989; Battista y Clements, 1995; Recio y Godino, 1996, Recio, 2000).
Pensamos, sin embargo, que los esfuerzos de clarificación realizados en el campo sobre la propia noción de demostración matemática, sobre sus distintas tipologías y relaciones mutuas, son insuficientes. La idea de demostración deductiva y formal, entendida de un modo rígido y absoluto en el seno de la comunidad matemática como la única concepción posible, parece necesitada de una revisión teórica. Consideramos, en este sentido, que es necesario realizar un estudio sistemático sobre los diversos significados de demostración en distintos contextos institucionales, así como sobre su relación con otras nociones próximas, tales como explicación, argumentación, razonamiento, verificación, etc. Este estudio 
podría facilitar la comparación de las aportaciones de distintas investigaciones, poner al descubierto nuevas cuestiones de investigación, aportar interpretaciones alternativas de las dificultades de los estudiantes y elaborar propuestas de intervención didáctica fundamentadas.

Creemos que los estudios sobre la demostración en educación matemática deben enmarcarse dentro de la problemática más amplia de la evaluación y desarrollo de las distintas prácticas argumentativas en diversos contextos institucionales. Sólo de esta manera seremos sensibles a las distintas modalidades de demostración, de sus condiciones de desarrollo, los papeles que desempeñan en los distintos contextos instituticionales y las relaciones entre las mismas.

En este trabajo vamos a analizar las diferencias de significado de la idea de demostración en distintos contextos institucionales, usando el marco teórico sobre los objetos matemáticos y sus significados desarrollado en Godino y Batanero (1994, 1998). En este caso implica adoptar las situaciones de validación y las prácticas argumentativas correspondientes como nociones de partida, de las cuales se derivan los diferentes tipos de demostración como emergente de los sistemas de prácticas argumentativas, distinguiendo, además, entre sus dimensiones institucionales y personales.

\section{SITUACIONES DE VALIDACIÓN Y PRÁC- TICAS ARGUMENTATIVAS}

La palabra demostración se utiliza en distintos contextos con diversos sentidos. A veces estos diversos sentidos y matices se reconocen mediante el uso de términos tales como explicación, argumentación, prueba, etc. Aunque en todos ellos se pueda reconocer una idea común -la de justificar o validar una afirmación (tesis) aportando razones o argumentos-; sin embargo, las diferencias en los tipos de situaciones en que se usan, sus rasgos característicos y los recursos expresivos puestos en juego en cada caso pueden ser, de hecho, diferentes. Estas diferencias en las situaciones y prácticas argumentativas indican sentidos distintos del concepto de demostración -o bien diversos objetos demostración según el modelo ontosemántico adoptado.

Balacheff (1987) utiliza el término explicación como idea primitiva de la cual deriva las de prueba y demostración. Para este autor, la explicación es un «discurso que pretende hacer inteligible el carácter de verdad, adquirido para el locutor, de una proposición o de un resultado». Balacheff propone reservar el término prueba para las «explicaciones aceptadas por una comunidad dada en un momento dado» (p. 148). Según esto, para que una explicación sea una prueba ha de ser vista o reconocida por alguien como razón suficiente en el correspondiente marco discursivo.

El uso que hace Balacheff de explicación difiere del de Duval (1993). Este autor distingue entre explicación y argumentación por el valor epistémico diferente de las razones aportadas en cada caso: «en la argumentación se trata de mostrar el carácter de verdad de una proposición, mientras que en la explicación los enunciados tienen una intención descriptiva de un fenómeno, resultado o comportamiento» (Duval, 1993, p. 40). Consideramos, no obstante, que la idea de explicación de Balacheff puede asimilarse a la noción de argumentación de Duval.

Balacheff y Duval usan el término demostración con significados similares: una secuencia de enunciados organizados según reglas determinadas. En matemáticas, un enunciado se asume convencionalmente como verdadero, o bien se deduce de los que le preceden con la ayuda de reglas de deducción tomadas de un conjunto de reglas bien definido cuya validez es socialmente compartida. Para Duval (1993), el objeto de la demostración es la verdad y, por tanto, obedece a criterios de validez, mientras que la argumentación se propone lograr la convicción del otro o de sí mismo, obedeciendo a criterios de pertinencia.

Una distinción importante es la que hace Krummeheuer (1995), siguiendo a Toulmin, entre argumentos analíticos y sustanciales. Los primeros son característicos de las deducciones lógicas correctas, siendo tautológicos, esto es, un aspecto latente de las premisas se elabora visiblemente, pero no añaden nada a la conclusión que no fuera ya una parte potencial de las premisas. Los argumentos sustanciales, por el contrario, expanden el significado de las proposiciones en la medida en que relacionan apropiadamente un caso específico a éstas por actualización, modificación o aplicación.

Desde un punto de vista cognitivo, las relaciones entre razonamiento y argumentación consideramos que son las que se establecen entre un constructo y sus indicadores empíricos. Balacheff (1987, p. 148) define el razonamiento como la «actividad intelectual, la mayor parte del tiempo no explícita, de manipulación de informaciones para producir nuevas informaciones a partir de datos». Desde nuestro punto de vista, esta actividad intelectual, que no puede reducirse meramente a la manipulación de informaciones, da origen a las prácticas argumentativas, personales o institucionales, que constituyen su dimensión ostensiva y comunicacional. Al mismo tiempo, el razonamiento se desarrolla por medio de dichas prácticas, de modo que el estudio del razonamiento está constitutivamente ligado al estudio de la argumentación.

En este trabajo utilizaremos el término demostración para referirnos de modo genérico al objeto emergente del sistema de prácticas argumentativas (o argumentos) aceptadas en el seno de una comunidad, o por una persona, ante situaciones de validación y decisión, esto es, situaciones que requieren justificar o validar el carácter de verdadero de un enunciado, su consistencia o la eficacia de una acción. Abarca, por tanto, las tres acepciones básicas de la verdad que reconoce la moderna teoría de la ciencia: correspondencia, consistencia y utilidad (Cañón, 1993).

En la siguiente sección trataremos de mostrar que la característica de una práctica discursiva de ser una de- 
mostración es un atributo esencialmente contextual y pragmático.

\section{SIGNIFICADOS DE LA DEMOSTRACIÓN EN DISTINTOS CONTEXTOS INSTITUCIO- NALES}

Desde un punto de vista cultural, Wilder (1981) nos recuerda que «no debemos olvidar que lo que constituye una "demostración" varía de una cultura a otra, así como de una época a otra» (p. 346). Nosotros vamos a tratar de mostrar que esta relatividad debe ampliarse a distintos contextos institucionales cuando nos interesamos por los problemas psicológicos y didácticos implicados en la enseñanza de la demostración.

Consideraremos que un contexto o marco institucional es un punto de vista local o una perspectiva sobre una problemática determinada, caracterizada por el uso de recursos expresivos e instrumentales propios, por hábitos y normas específicas de comportamiento. En lo que sigue trataremos de mostrar la diversidad de demostraciones según los contextos institucionales siguientes: lógica y fundamentos de las matemáticas, matemática profesional, vida cotidiana, ciencias experimentales y enseñanza de las matemáticas elementales (incluyendo aquí los niveles primarios, secundarios y universitarios). En cada uno de estos contextos es posible identificar, a su vez, puntos de vista más locales en los cuales los problemas de la verdad y la demostración adquieren connotaciones específicas. Así, por ejemplo, las principales tendencias sobre los fundamentos de las matemáticas (logicismo, formalismo, intuicionismo, cuasi empirismo) mantienen visiones diferentes sobre el papel de la demostración en matemáticas y sobre los criterios de validez de una demostración matemática (Hanna, 1995, p. 42). Sin embargo, consideramos que el nivel de análisis que hemos adoptado en este trabajo es suficiente para mostrar la diversidad de objetos identificables y, en particular, que no hay una teoría y una práctica uniforme, firmemente establecida, sobre la demostración matemática.

\section{Lógica y fundamentos de las matemáticas}

En lógica y fundamentos de las matemáticas (al menos en las corrientes preponderantes), la noción de demostración está íntimamente ligada a las nociones de deducción y de sistema axiomático (o formal). Como afirma Garrido (1978), el argumento deductivo si no es el único, es el principal objeto de la lógica formal, usándose la palabra argumento como sinónimo de argumento deductivo. Los argumentos deductivos se realizan en el seno de un lenguaje simbólico que se construye a partir de una serie de símbolos elementales y de unas reglas de formación de fórmulas, de expresiones precisas, perfectamente definidas y partiendo de dichos símbolos. Ese lenguaje formal es la base de un sistema axiomático en el que las deducciones se llevan a cabo. Lo característico de un sistema axiomático es disponer de un conjunto de enunciados que se admiten sin prueba y a partir de los cuales se obtienen todos los demás enunciados de la teoría, mediante un conjunto preciso de reglas de transformación.

En estos contextos, la veracidad de un teorema descansa en la validez de las reglas lógicas usadas en el proceso de demostración. El teorema aparece como una consecuencia lógica y necesaria de las premisas de que parte, mediante la correspondiente inferencia deductiva. Un enunciado (o teorema) aceptado como verdadero tiene una validez universal e intemporal garantizada por la validez de las reglas lógicas usadas en la prueba.

Interesa también resaltar la índole de las situaciones problemáticas que se afrontan en estos contextos. Se trata de justificar, con las máximas garantías, la verdad del sistema de proposiciones matemáticas o, al menos, una parte del mismo. Esto implica buscar el sistema mínimo de axiomas (verdades evidentes por sí mismas), independientes entre sí, no contradictorio y completo, de tal manera que, aplicando las reglas de inferencia lógica, puedan derivarse el resto de las proposiciones matemáticas. Hay, pues, una problemática teórica de organización y estructuración del cuerpo de conocimientos matemáticos. Para realizar este trabajo con las máximas garantías y rigor se requiere el uso de lenguajes formales.

El objeto «demostración» en estos contextos institucionales podemos describirlo sintéticamente como emergente del sistema de prácticas argumentativas analíticas formales, y su significado viene dado por los rasgos intensionales, extensionales y representacionales descritos.

Esto no significa, no obstante, que incluso en estos contextos institucionales no se utilizen argumentaciones de tipo sustancial para justificar afirmaciones. La aceptación de los axiomas o postulados de cualquier sistema matemático se hace necesariamente mediante argumentaciones intrínsecamente inductivas. Recordemos a este respecto lo que nos dice Poincaré (1902): «¿Cuál es la naturaleza del razonamiento matemático? ¿Es realmente deductivo como ordinariamente se cree? Un análisis profundo nos muestra que no es así; que participa en una cierta medida de la naturaleza del razonamiento inductivo, y que por eso es fecundo.» (p. 15)

\section{Origen de la necesidad de los teoremas matemáticos}

Las ideas de Wittgenstein sobre la demostración difieren sustancialmente de las defendidas por los restantes filósofos interesados por los fundamentos de las matemáticas al introducir un componente convencionalista en el núcleo de la misma. Para Wittgenstein, la necesidad lógica de cualquier enunciado es siempre la expresión directa de una convención lingüística. Un teorema matemático no expresa una propiedad universal e intemporal de ciertas entidades ideales; se trata, en cambio, de la expresión disfrazada de una regla aceptada que establece lo que se debe obtener cuando los procesos de cálculo 
pertinentes se realizan correctamente. La demostración es concebida como una representación paradigmática de la correcta realización de ciertas transformaciones de signos: «La demostración es nuestro modelo de un determinado resultado, que sirve de objeto de comparación (patrón) para trasformaciones reales.» (Wittgenstein, 1978, p. 132)

Su función es persuadir de que conviene extender el aparato conceptual en una cierta dirección, de que es útil aceptar la regla gramatical expresada en el enunciado del teorema. Para que una cierta construcción de signos -un dibujo geométrico, una imagen formada por numerales dispuestos de un cierto modo, o incluso una larga secuencia de sentencias escritas- sea una demostración matemáticas, se debe adoptar la decisión de aceptar ciertas definiciones previas de los elementos que intervienen, una decisión que es equivalente a atribuir a la construcción de signos en cuestión el papel de paradigma de ese procedimiento correcto.

Wittgenstein no niega que las proposiciones matemáticas condicionales del tipo «si uno aplica correctamente la regla "Suma 2" a 1000, obtiene 1002» tengan un carácter necesario. La peculiaridad de su posición - derivada de sus interpretaciones del seguimiento de una regla-se refiere a la naturaleza de la necesidad: la fuente de la necesidad es una decisión lingüística que declara que no tiene sentido cualquier enunciado empírico que identifica un número distinto de 1002 como el resultado producido, en una cierta ocasión, por una correcta aplicación de la regla «Suma 2» a 1000. (Frascolla, 1994, p. 128). La aceptación de la proposición $2+2=4$ con carácter necesario e intemporal no es porque se corresponda con propiedades de objetos ideales -que por ello expresa una verdad universal-, sino porque hemos sido entrenados en seguir unas reglas de uso (definiciones) de los símbolos $2,+, 4$.

Queda abierta la cuestión de cuáles deberían ser las consecuencias de esta filosofía sobre la práctica de la enseñanza y el aprendizaje de las matemáticas, pero intuimos que pueden ser cruciales.

\section{Matemática profesional}

La noción de demostración difiere fuertemente desde el punto de vista de la lógica formal y estudios fundacionales matemáticos respecto a la práctica matemática real. Como indica Otte (1994), la visión lógica de la demostración pone el acento en los aspectos lingüísticos y formales. Intentando liberar la demostración de contextos concretos prescinde del significado de las expresiones del sistema y las reduce a expresiones sintácticas, sujetas a reglas de transformación formales. Reglas que actúan sobre cadenas de texto, transformándolas en otras cadenas de texto, que ejercen en todo como si tales cadenas carecieran de significado. La matemática, desde esta concepción, se transforma en una gramática formal. La demostración se convierte en un procedimiento puramente algorítmico, que puede ser materializado mediante el uso de ordenadores.
Las demostraciones, bajo este esquema, se tornan extraordinariamente complejas, lo que hace que, para muchas investigaciones matemáticas, la formalización completa de las pruebas, incluso aunque fuera posible en principio, se hace imposible en la práctica. «Pueden requerir tiempo, paciencia e interés más allá de la capacidad de cualquier matemático humano. Ciertamente, pueden exceder la capacidad de cualquier sistema de computación disponible o previsible.» (Hersh, 1993, p. 390). Livington (1987), por ejemplo, muestra claramente la complejidad de la demostración formalizada de la unicidad del elemento neutro en teoría de grupos $\left(\mathrm{e}=\mathrm{e}^{*} \mathrm{e}^{\prime}=\mathrm{e}^{\prime}\right)$, comparada con su trivialidad mediante una argumentación analítica informal.

Esto hace, como afirma Resnick (1992), que la matemática contemporánea esté repleta de working proofs, esto es, demostraciones informales, no axiomatizadas. «Incluso un campo metodológicamente tan consciente como la lógica matemática se desarrolló de modo no axiomático, y en su enseñanza los lógicos la presentan como una colección de pruebas y teoremas informales» (p. 12).

En el ámbito profesional matemático, las demostraciones son deductivas pero no formales, se expresan mediante el lenguaje ordinario completado con el uso de expresiones simbólicas. La fundamentación de una inferencia $\mathrm{A} \Rightarrow \mathrm{B}$ no estriba en la existencia de una regla de transformación que permite pasar de A a B, sino que se apoya en la significación particular de las expresiones A y $\mathrm{B}$, interpretada por el matemático que realiza la inferencia. De este modo, los teoremas matemáticos pierden de hecho su carácter de verdades absolutas, necesariamente verdaderas (falibilismo).

Desde la propia matemática están surgiendo, recientemente, nuevas estrategias de validación de las proposiciones matemáticas que desafían la concepción clásica de la demostración deductiva línea a línea, tales como la zero-knowledge proof, holographic proof, visual proof, $\mathrm{y}$ en general las pruebas basadas en comprobaciones experimentales, a menudo en el uso de ordenadores (Hanna, 1995, p. 43).

Así pues, en este contexto, y en el de la «nueva epistemología de la matemática» (Lakatos, Davis y Hersh, Ernest, Tymoczko, etc.), la demostración adquiere rasgos distintos. Ante la imposibilidad de aplicar los métodos formales de la lógica matemática a la demostración de cualquier proposición matemática, la verdad de las proposiciones matemáticas avanzadas no queda plenamente garantizada. Por la gran dificultad y especialización de los temas tratados en la matemática profesional, no puede afirmarse la verdad definitiva de los teoremas publicados. De este modo, la matemática real adquiere un carácter falibilista, social, convencional, temporal. Esta situación lleva a Hersh (1993, p. 389) a describir la demostración en la práctica real del matemático como «un argumento convincente juzgado como tal por jueces cualificados». No hay un estándar generalmente aceptado del grado de rigor y sistematización exigible a una demostración matemática; esto es, existen diversos tipos de justificaciones, aunque probablemente 
debamos reconocer entre ellos algún tipo de organización jerárquica.

Como señala Hanna (1989), la concepción tradicional bourbakista, basada en una visión formal, abstracta y rigurosa de las matemáticas, está cambiando. «Durante las dos últimas décadas varios matemáticos y educadores matemáticos han desafiado el principio de que el aspecto más significativo de las matemáticas sea el razonamiento por deducción, que culmina en pruebas formales. Desde su punto de vista hay mucho más en las matemáticas que sistemas formales. Este punto de vista reconoce la realidad de la práctica matemática. Los matemáticos admiten que su demostración puede tener diferentes grados de validez formal -y todavía adquirir el mismo grado de aceptación. Los matemáticos están de acuerdo además en que, cuando una demostración es válida en virtud de su forma sólo, sin atender a su contenido, es probable que añada muy poco a la comprensión de su materia e irónicamente puede no ser muy convincente.» (Hanna, 1989, p. 20).

En cuanto a la problemática afrontada por el matemático profesional, se centra en la resolución de nuevos problemas, en incrementar el cuerpo de conocimientos y, en menor medida, en la organización y fundamentación del sistema completo de las matemáticas. No se requiere el grado de máxima seguridad características del trabajo realizado por las personas interesadas por los fundamentos de las matemáticas. Balacheff (1987) distingue tres tipos de situaciones que reclaman procesos de validación, las cuales son reconocibles también en el trabajo del matemático. Este autor caracteriza estas situaciones por la función de las demostraciones producidas: para decidir, convencer y saber. En las dos primeras, el criterio principal es la eficacia, mientras que en la tercera es el del rigor.

\section{Ciencias experimentales}

La demostración, en estos contextos, se basa principalmente en prácticas argumentativas de tipo sustancial (empírico inductivas, analógicas, etc.) por medio de las cuales concluimos que lo que es verdadero de ciertos individuos de una clase es verdadero de toda la clase o bien que lo que es verdadero, algunas veces, lo será siempre en circuntancias semejantes o con una cierta probabilidad. No descarta el empleo simultáneo de argumentaciones deductivas, en particular inferencias estadísticas. La validez de los enunciados de contenido empírico:

a) no tiene carácter absoluto y universal;

b) su validez se incrementa a medida que se muestran o producen más hechos que se ajusten al enunciado;

c) un ejemplo que no se cumpla no invalida completamente la afirmación.

La demostración pone en juego los recursos expresivos de los lenguajes ordinario, simbólico y cualquier tipo de dispositivos concretos. En el caso de las ciencias expe- rimentales, los experimentos u observaciones se hacen con el máximo cuidado, controlando todos los factores posibles que pueden influir en el mismo.

La argumentación matemática presenta, en los dominios científicos, connotaciones de prueba empírica. Las teorías matemáticas son consideradas verdaderas porque, independientemente de sus interrelaciones formales y deductivas, pueden ser comprobadas de forma experimental en situaciones fenomenológicas variadas. Para autores como Kline (1980, p. 400): «La corrección de las matemáticas debe ser juzgada por su aplicabilidad al mundo físico [...] Son correctas en la medida que funcionan y, cuando no funcionan, deben ser modificadas.» Es, según esta interpretación, su utilidad para fundamentar teorías científicas consolidadas lo que prueba, en último extremo, la validez de las teorías matemáticas.

\section{La verificación en ciencias y matemáticas}

Granger (1992) estudia la función y el valor de la verificación para la construcción del conocimiento científico en general, así como su papel auxiliar en la demostración matemática. La verificación presenta en matemáticas una función paradójica en cierto sentido, ya que los teoremas matemáticos son por naturaleza demostrados y verdades de carácter general; la verificación, por el contrario, parece aplicarse a enunciados tomados en su particularidad. Granger, sin embargo, analiza las situaciones en las cuales verificar equivale a demostrar -donde la verificación de un enunciado coincide con la explicitación de su mismo sentido-y cuando desempeña un papel de auxiliar de la demostración. Elabora una tipología simple de la verificación en matemática, distinguiendo tres tipos:

1) Verificación de un resultado de operación simple, como, por ejemplo, los enunciados de la aritmética elemental. ¿Qué quiere decir verificar que $7+5=12$ ?

Un primer sentido obvio consiste en proyectar los objetos abstractos en lo empírico, y en comprobar empíricamente un resultado, independientemente de la especificación material, sensible, de los objetos elegidos como proyecciones de los abstractos. Las entidades abstractas son aquí los números, dotadas a la vez de un estatuto de objetos y de un estatuto operatorio. La comprobación inmediata de la adecuación de un esquema operatorio y de los objetos abstractos, o representados concretamente, es un privilegio de la aritmética elemental, que se basa en la posibilidad de una exploración en un número finito de pasos del enunciado a verificar. En este caso, «verificar significa iterar de manera finita una operación elemental y reconocer los objetos así producidos correlativamente» (p. 91).

2) Un segundo tipo de verificación aparece cuando las operaciones cuyo resultado es el enunciado a verificar presentan un cierto nivel de complejidad técnica. Aunque también en este caso verificar consiste en iterar una operación elemental un número finito de veces, este número puede ser demasiado grande para que la iteración sea efectiva. La naturaleza de los mismos objetos y 
operaciones que intervienen en el enunciado determina el tipo de verificación puesta en juego. En esta segunda especie, la verificación es un cálculo cuya realización efectiva puede verse afectada por posibles errores y, por tanto, no proporciona sino una garantía parcial de la verdad o falsedad del enunciado.

3) El tercer tipo de verificación se refiere a los enunciados relativos a construcciones geométricas. ¿Qué es verificar un enunciado de geometría representado en una figura? Como en los casos precedentes, afirma Granger, consiste en efectuar operaciones, real o virtualmente. Pero su resultado depende, entonces, en general de un contenido ligado a lo empírico. Se trata en este caso de proyectar en lo empírico los conceptos abstractos y las operaciones sobre estos conceptos.

Para los enunciados aritméticos elementales, esta proyección hace posible un procedimiento de verificación completa, como consecuencia de la finitud de los conjuntos de elementos explorados, pero también por su carácter «discreto». «Los objetos geométricos para los que se propone verificar propiedades no poseen sino excepcionalmente finitud del número de elementos que se consideran en ellos, ni carácter discreto. Los procedimientos de verificación dejan de tener en este caso la claridad y el carácter decisorio que tenían en las dos especies precedentes.» (p. 94) En este caso es cuando una actividad demostrativa se inserta sobre los propios procesos de verificación, los cuales adquieren una función de auxiliar de la demostración matemática.

En síntesis, la naturaleza de la verificación y sus relaciones con la demostración son, según Granger, las siguientes (p. 113):

a) Verificar en matemáticas es comprobar un resultado de operación, simple o compleja, realizada, en definitiva, sobre símbolos.

b) La condición determinante de la autonomía de la verificación es la exhaustividad de los campos operatorios. Cuando esa exhaustividad falla, los enunciados matemáticos no tienen sino un sentido que depende, entonces, de demostraciones.

c) El estatuto de la verificación, y su validez misma, depende de manera esencial de la naturaleza de los objetos que aparecen en los enunciados sometidos a verificación.

Los procesos de verificación están, por tanto, estrechamente asociados a tentativas de demostración, coronadas o no por el éxito, desempeñando un papel heurístico y exploratorio (por ejemplo, para rechazar una conjetura), aunque insuficiente, excepto en la verificación de resultados de operaciones simples.

\section{Vida cotidiana}

En la vida cotidiana se suele usar una argumentación informal, que es situacional, dependiente del contexto e incluso dependiente de la propia situación emocional del sujeto. Este tipo de argumentación informal no da lugar necesariamente a verdades, porque se apoya en consideraciones que pueden tener exclusivamente un valor local, careciendo del valor objetivo de la prueba.

En el razonamiento natural o informal, puesto de manifiesto en nuestras inferencias cotidianas, desempeña un papel importante el razonamiento por analogía. Toda inferencia analógica parte de la similitud de dos o más cosas en uno o más aspectos para concluir la similaridad de esas cosas en algún otro aspecto. El razonamiento informal implica razonamiento sobre las causas y las consecuencias, y sobre las ventajas y desventajas, los pros y los contras de determinadas proposiciones o de alternativas sobre las que hay que decidir. Fernández y Carretero (1995, p. 43) atribuyen a la lógica informal las siguientes características:

- Se aplica a cuestiones de la vida cotidiana, aunque también a cuestiones profesionales o académicas.

- Se aplica a cuestiones relevantes para el individuo.

- Está relacionada con la capacidad de elaborar y valorar argumentos y contraargumentos.

- No utiliza un lenguaje formal o simbólico sino el lenguaje ordinario.

- Es dinámico y muy dependiente del contexto.

- Se aplica a tareas abiertas y mal definidas.

- Se aplica a tareas no deductivas.

El razonamiento informal es utilizado en todos los dominios de conocimiento, incluso en las matemáticas y las ciencias naturales. Si, como afirman Garuti, Boero y Lemut (1998, p. 345), hay continuidad cognitiva entre la fase de producción de conjeturas y la construcción de demostraciones, puede considerarse que estas formas argumentativas constituyen, en matemáticas, los primeros estadios de la demostración.

\section{La demostración en la clase de matemáticas}

Para los currículos, textos y profesores de matemáticas de los niveles de enseñanza primaria, secundaria y universitaria, en general, los teoremas matemáticos son necesariamente verdaderos. Pero las argumentaciones que establecen esa verdad son, en el mejor de los casos, argumentaciones deductivas informales y con frecuencia argumentaciones no deductivas o incluso argumentaciones basadas en criterios externos de autoridad.

La matemática elemental -incluida la que se enseña en los cursos universitarios- constituye un cuerpo de conocimiento cuya verdad, en general, no se pone en duda. Se disponen de distintas pruebas para los teoremas que son aceptadas por la generalidad de los matemáticos profesionales. Constituye, pues, un cuerpo de conocimientos exento del carácter falibilista que se atribuye a algunos resultados de la «matemática avanzada»o, al menos, así se suele presentar en los textos correspondientes y en las clases de matemáticas. «La epistemología escolar es fundamentalmente platonista; tanto los conocimientos matemáticos como los criterios de validez de un enun- 
ciado preexisten a cualquier suceso que pudiera ocurrir en la clase.» (Balacheff, 1987, p. 172)

Herbst (1998) ha observado, desde una perspectiva naturalista, distintos modos de impartir clases de matemáticas de nivel secundario analizando cómo funciona la demostración en dichas clases, cómo se valida en ellas el conocimiento matemático y qué fórmulas de negociación establecen el profesor y los alumnos al respecto. Identificó nueve categorías de prácticas de validación efectivamente usadas: el ejemplo genérico, el ejemplo aislado, los experimentos cruciales, la ostensión como prueba, la justificación pseudomatemática, la analogía y la metáfora, la simetría u ostensión metafórica, el cálculo simbólico y la prueba «oficial».

En estos contextos institucionales, particularmente en los niveles superiores, se espera de los estudiantes que adquieran la capacidad de comprender y realizar pruebas de teoremas matemáticos, que establezcan la verdad de los mismos con absoluta seguridad, convenciéndose a sí mismos y a cualquier persona de dicha verdad de manera irrefutable. Se trata de un uso de la demostración idiosincrásico, distinto del que se hace por el matemático profesional. Éste tiene que elaborar demostraciones que convenzan a los referees de las revistas; el estudiante de matemáticas tiene que convencerse, y explicar su convencimiento al profesor, de la verdad necesaria y universal del teorema.

Como afirma Balacheff (1987), en la práctica profesional de la investigación matemática, una demostración es una demostración hasta que se demuestra lo contrario. Pero esta posición es difícil de mantener en el contexto escolar. «En un momento u otro, los alumnos, puesto que "están allí para aprender", tienen necesidad de una garantía sobre la validez de sus producciones y, si existen posiciones contradictorias en la clase, no pueden subsistir juntas durante mucho tiempo.» (p. 173). El profesor tiene que sancionar la verdad o la falsedad de los enunciados, la corrección de las pruebas y las interpretaciones producidas en la clase, lo que condiciona y determina el estatuto de la verdad y la demostración en este contexto institucional.

\section{SIGNIFICADOS PERSONALES DE LA DE- MOSTRACIÓN MATEMÁTICA}

En la literatura de investigación en educación matemática abundan las publicaciones que describen las dificultades de los estudiantes de los distintos niveles educativos con las demostraciones matemáticas, así como sus modos específicos de razonamiento.

Harel y Sowder (1996) han estudiado la dimensión subjetiva o personal de la demostración. Estos autores, al proceso empleado por un individuo para suprimir o apartar dudas sobre la verdad de una conjetura lo llaman demostración. «Un esquema de demostración individual consiste en lo que constituye asegurar y persuadir para esa persona» (pp. 3-60). De manera equivalente pode- mos interpretar dichos esquemas como «lo que significa demostrar» para un sujeto, esto es, el significado personal del objeto demostración según proponen Godino y Batanero (1994,1998). Las distintas categorías de esquemas de demostración que identifican Harel y Sowder representan un estadio cognitivo, una habilidad intelectual en el desarrollo matemático de los estudiantes, y son derivadas de las acciones realizadas por los sujetos en procesos de demostración. Distinguen tres categorías principales de esquemas de demostración: basados en convicciones externas (ritual, autoritario y simbólico), empíricos (inductivos y perceptuales) y analíticos (transformacionales y axiomáticos).

Para Harel y Sowder, la alta incidencia entre los estudiantes de los tres subtipos de esquemas de demostración basados en «convicciones externas» así como el esquema de demostración empírico-inductivo pueden explicarse por la influencia de hábitos escolares en consonancia con tales tipos de prácticas argumentativas. El análisis que hemos presentado en las secciones anteriores sugiere, en efecto, que en las clases de matemáticas elementales pueden predominar las prácticas argumentativas no analíticas, sobre todo en los niveles de enseñanza primaria y secundaria. Estas prácticas argumentativas -implementadas de manera inconsciente por los profesores de matemáticas- pueden haber sido extrapoladas desde otros contextos institucionales, como los de la vida cotidiana o de las ciencias empíricas.

No se debe olvidar además el papel que juegan las formas argumentativas sustanciales en las fases de búsqueda y formulación de conjeturas en la resolución de problemas. Los argumentos analíticos, propios de la demostración matemática, no son las únicas prácticas argumentativas que utiliza el matemático profesional para convencerse de la verdad de sus conjeturas. Este modo de razonar se revela con frecuencia estéril, cuando no un estorbo, en las fases de creación/descubrimiento de las soluciones de los problemas, en las cuales es lícito y necesario desplegar formas de argumentación sustanciales, en particular, la inducción empírica y la analogía. Recordemos las palabras de Polya (1944, p. 116): «Las matemáticas presentadas con rigor son una ciencia sistemática, deductiva, pero las mátemáticas en gestación son una ciencia experimental, inductiva.»

\section{CONCLUSIONES E IMPLICACIONES PA- RA LA INVESTIGACIÓN Y LA ENSEÑANZA}

Sin duda podemos apreciar algunos rasgos comunes en los usos de la palabra demostración en los distintos contextos institucionales descritos, lo que permite hablar de la idea de demostración en sentido general. Pero este modo de hablar genérico, abstracto, metafísico no debe ocultar la rica y compleja variedad de sentidos que adquiere el concepto de demostración o, si se prefiere, la diversidad de «objetos demostración» existentes para los miembros de dichas instituciones, cada uno con un significado local. Creemos que, cuando estamos interesados por los problemas psicológicos y didácticos invo- 
lucrados en los procesos de validación de proposiciones matemáticas, interesa considerar que no hay un concepto de demostración sino diversos, tanto desde el punto de vista subjetivo como institucional (Godino y Batanero, 1994, 1998).

Reconociendo esta diversidad de objetos y significados, estaremos en mejores condiciones de estudiar los componentes del significado, las circunstancias de su desarrollo, los papeles que desempeñan en los distintos contextos, en definitiva, de comprender las relaciones ecológicas que se establecen entre los mismos y su carácter sistémico. Esta modelización ontosemántica, de carácter antropológico, puede ayudar a tomar conciencia de los conflictos cognitivos y culturales que se plantean a todo individuo que se ve forzado a participar como sujeto en distintos contextos institucionales.

Dado que los estudiantes se encuentran simultáneamente sujetos a distintas instituciones, en cuyo seno se ponen en práctica distintos esquemas argumentativos, parece razonable que los estudiantes tengan dificultades en discriminar el uso respectivo de cada tipo de argumentación. En consecuencia, consideramos que tales esquemas institucionales de demostración pueden ser factores explicativos de los esquemas subjetivos manifestados, por lo que deben ser tenidos en cuenta e investigados con más profundidad.

En el propio y genuino trabajo del matemático profesional conviven las distintas formas básicas de razonamiento: el empírico-inductivo (argumentación sustancial, prueba empírica) y el lógico-deductivo (en sus distintas modalidades, transformacionales y axiomáticas). Ambas formas están relacionadas con distintas fases del proceso de matematización. La primera entra en juego básicamente en las fases de creación/ descubrimiento de las soluciones de un problema, mientras que la segunda, en los procesos de formulación, comunicación y validación frente a otras personas (y frente a uno mismo). En los distintos niveles de enseñanza se precisa articular de algún modo los distintos significados de la prueba, desarrollando progresivamente en los estudiantes los conocimientos, la capacidad discriminativa y la racionalidad que se debe poner en juego en cada caso. Los esquemas informales de demostración no pueden ser vistos simplemente como incorreciones, errores o deficiencias, sino como etapas en la apropiación y dominio de las prácticas argumentativas matemáticas.

La enseñanza de las matemáticas debe procurar que los estudiantes controlen y dominen las diversas prácticas argumentativas, así como ser conscientes de las relaciones dialécticas entre las mismas. No obstante, la complejidad del significado de los argumentos analíticos en el contexto de la matemática profesional implica la parcialidad de su comprensión y el dominio en los niveles de secundaria e, incluso, universitaria.

La enseñanza de la argumentación analítica debe quedar integrada como una fase más del quehacer matemático, en la que se trata de convencer a uno mismo y a los demás del carácter necesariamente verdadero de las conjeturas matemáticas. Pero esta fase tiene que incardinarse con el planteamiento de las cuestiones, la elaboración de suposiciones y argumentos heurísticos potencialmente verdaderos, como propone la teoría de situaciones de Brousseau (1986). De lo contrario, se corre el riesgo de forjar en el estudiante una visión reduccionista e irreal, aunque popular, de las matemáticas, según la cual ésta es una colección de definiciones, teoremas y pruebas.

Estamos de acuerdo con Schoenfeld (1994, p. 76) cuando afirma que «la demostración no es una cosa separable de las matemáticas, tal y como aparece en los currículos; es un componente esencial del hacer, comunicar, y registrar las matemáticas. Y pienso que puede ser incorporada en nuestros currículos, en todos los niveles.» (p. 76)

Pero también debemos reconocer que los procesos de validación -y, por tanto, las demostraciones- puestos en práctica en los niveles de educación primaria serán distintos de los realizados en secundaria y éstos diferentes a su vez de los universitarios. «Es necesario tomar en consideración la naturaleza de la racionalidad de los alumnos y las condiciones de su evolución, pero también tomar en cuenta el análisis didáctico de los criterios aceptados de demostración que deben poder evolucionar en el curso de la escolaridad.» (Balacheff, 1987, p. 170)

La comprensión y el dominio de la argumentación axiomático-deductiva por parte de los estudiantes requiere el desarrollo de una racionalidad y un estado específico de los conocimientos. Exige «la adhesión a una problemática que no es la de la eficacia (exigencia de la práctica) sino la del rigor (exigencia teórica) (Balacheff, 1987, p. 170). En esta misma idea incide Fischbein (1982) cuando afirma que, para la comprensión de lo que realmente significa una demostración matemática, «la mente del alumno debe sufrir una modificación fundamental» (p. 17). Pero la construcción de esta racionalidad es un proceso progresivo que requiere tiempo, así como adaptaciones ecológicas del «objeto prueba» (transposiciones didácticas) en los distintos niveles de enseñanza.

Somos conscientes de que se precisa realizar nuevas investigaciones para identificar con más detalle las características de las diversas variedades de demostración esbozadas en este trabajo en los distintos contextos institucionales considerados. En particular, es necesario realizar investigaciones empíricas, mediante observaciones etnográficas de clases y entrevistas clínicas a estudiantes, para verificar la principal hipótesis que ha emergido de nuestra indagación: «que los esquemas institucionales de demostración son variables explicativas de los esquemas personales y que los conflictos en el uso de dichos esquemas son motivados por la superposición indiscriminada de las diversas formas argumentativas en la actividad matemática de la clase».

No pretendemos que de nuestras reflexiones se puedan derivar normas sobre cómo afrontar el tema de la demostración en la práctica de la enseñanza de las matemáticas. No obstante, una consecuencia razonable de este trabajo 
para la práctica docente que podemos hacer se puede expresar en los siguientes términos: «es necesario que los profesores de matemáticas sean conscientes de la variedad de argumentaciones usadas por las personas para validar sus afirmaciones según los contextos institucionales en que se hacen tales afirmaciones y de las diversas formas argumentativas que se ponen en juego en la clase de matemáticas». El problema de cómo lograr que los alumnos dominen las diversas técnicas argumentativas y discriminen su uso según las circunstancias de la tarea solicitada debe ser objeto de reflexión e investi-

\section{REFERENCIAS BIBLIOGRÁFICAS}

BALACHEFF, N. (1987). Processus de preuve et situations de validation. Educational Studies in Mathematics, 18, pp. 147-176.

BATTISTA, M.T. y CLEMENTS, D.H. (1995). Geometry and proof. The Mathematics Teacher, 88(1), pp. 48-54.

BROUSSEAU, G. (1986). Fondements et méthodes de la didactique des mathématiques. Recherches en Didactique des Mathématiques, 7(2), pp. 33-115.

CAÑÓN, C. (1993). La matemática: creación o descubrimiento Madrid: Universidad Pontificia de Comillas.

DUVAL,R.(1993). Argumenter, demontrer, expliquer: continuité ou rupture cognitive? Petit $x, 31$, pp. 37-61.

FERNÁNDEZ, P. y CARRETERO, M. (1995). Perspectivas actuales en el estudio del razonamiento, en Carreter, M., Almaraz, J. y Fernández, P. (eds.). Razonamiento y comprensión. Madrid: Trotta.

FISCHBEIN, E. (1982). Intuition and proof. For the learning of mathematics, 3(2), pp. 9-24.

FRASCOLLA, (1994). Wittgenstein's philosophy ofmathematics. Londres: Routledge.

GARRIDO, M. (1978). Lógica simbólica. Madrid: Tecnos.

GARUTI, R., BOERO, P. y LEMUT. E. (1998). Cognitive units of theorems and dificulty of proof, en Olivier, A. y Newstead, K. (eds.). Proceedings of the 22nd Conference of the International Group for The Psychology of Mathematics Education, vol. 2, pp. 345-352. Stellenbosch (Suráfrica): University of Stellenbosch.

GODINO,J.D. y BATANERO,C. (1994). Significadoinstitucional y personal de los objetos matemáticos. Recherche en Didactique des Mathématiques, 14(3), pp. 325-355.

GODINO, J.D. y BATANERO, C. (1998). Clarifying the meaning of mathematical objects as a priority area of research in mathematics education, en Sierpinska, A. y Kilpatrick, J. gación, configurando una línea de investigación en educación matemática a la que se están dedicando importantes esfuerzos.

\section{NOTA}

Este artículo es una versión revisada y ampliada del research report publicado en las actas del XXI PME celebrado en Lahti, (Finlandia) en 1997, con el título «Meanings of proof in mathematics education». (eds.). Mathematics education as a research domain: $A$ search for identity (pp. 177-195). Dordrecht: Kluwer, A.P.

GRANGER, G.G. (1992). La vérification. París: Odile Jacob.

HANNA, G. (1989). Proofs that prove and proofs that explain, en Vergnaud, G., Rogalski, J. y Artigue, M. (eds.). Proceedings of the 13th Conference of the International Group for the Psychology of Mathematics Education, pp. 45-54. París.

HANNA, G. (1995). Challenges to the importance of proof. For the Learning of Mathematics, 15(3), pp. 42-49.

HAREL, G. y SOWDER, L. (1996). Classifying processes of proving, en Puig, L. y Gutiérrez, A. (eds.). Proceedings of the 20th Conference of the International Group for the Psychology of Mathematics Education, vol. 3, pp. 59-65. Valencia: Universitat de València.

HERBST, P. (1998). «What works as proof in the mathematics class?» Athens (EEUU): University of Georgia. Tesis doctoral.

HERSH, R. (1993). Proving is convincing and explaining. Educational Studies in Mathematics 24, pp. 389-399.

KLINE, M. (1980). Matemáticas. La pérdida de la certidumbre. Madrid: Siglo XXI [1985].

KRUMMEHEUER, G.(1995). The ethnography of argumentation, en Cobb, P. y Bauersfeld, $\mathrm{H}$. (eds.). The emergence of mathematical meaning. Hillsdale, Nueva Jersey: Lawrence Erlbaum Associates.

LESTER, F.K. (1975). Developmental aspects of children's ability to understand mathematical proof. Journalfor Research in Mathematics Education, pp. 14-25

LIVINGSTON (1987). The ethnomethodological foundations of mathematics. Londres: Routledge \& Kegan Paul.

MARTIN, W.G. y HAREL, G. (1989). Proof frames of preservice elementary teachers. Journal for Research in Mathematics Education, 20(1), pp. 41-51. 
OTTE, M. (1994). Mathematical knowledge and the problem of proof. Educational Studies in Mathematics 26, pp. 299-321.

POINCARÉ, H. (1902). La science et l'hipothèse. Trad. de Besio, A.B. y Banfi, J. 1963. La ciencia y la hipótesis. Madrid: Espasa-Calpe.

POLYA, G. (1944). How to solve it. Priceton: University Press. Trad. cast. 1985. Cómo plantear y resolver problemas. México: Trillas.

RECIO, A.M. (1999). «Una aproximación epistemológica a la enseñanza y el aprendizaje de la demostración matemática». Granada: Departamento de Didáctica de la Matemática, Universidad de Granada. Tesis doctoral.

RECIO, A.M. y GODINO, J.D. (1996). Assessment of university students' mathematical generalization and symbolization capacities, en Puig, L. y Gutiérrez, A. (eds.). Proceedings of the 20th Conference of the International Group for the
Psychology of Mathematics Education (pp. I-231). Valencia: Universitat de València.

RESNICK, M.D. (1992). Proof as a source of truth, en Detlefsen, M. (ed.). Proof and knowledge in mathematics, pp. 6-32. Londres: Routledge.

SCHOENFELD, A.H. (1994). What do we know about mathematics curricula? Journal for Mathematical Behaviour, 13 , pp. 55-80

SENK, S.L. (1985). How well do students write geometry proofs? Mathematics Teacher. Sep. 1995, pp. 448-456.

WILDER, R.W. (1981). Mathematics as a cultural system. Nueva York: Pergamon.

WITTGENSTEIN, L. (1978). Remarks on the foundations of mathematics (3a. ed.). Oxford: Basil Blackwell. Trad. cast. 1987. Madrid: Alianza.

[Artículo recibido en julio de 2000 y aceptado en mayo de 2001.] 